\title{
Influence on Corporate Performance by Determinants of Working Capital
}

\author{
Roslin Lazarus ${ }^{1, *}$, Stanley Lazarus $^{2}$, Shobhna Gupta ${ }^{1}$ \\ ${ }^{1}$ Professional Studies and Undergraduate Department, College of Banking and Financial Studies, Muscat, Sultanate of Oman \\ ${ }^{2}$ Independent Researcher-Student, Monash University, Australia
}

Received March 23, 2021; Revised May 23, 2021; Accepted June 4, 2021

\section{Cite This Paper in the following Citation Styles}

(a): [1] Roslin Lazarus, Stanley Lazarus, Shobhna Gupta, "Influence on Corporate Performance by Determinants of Working Capital," Universal Journal of Accounting and Finance, Vol. 9, No. 3, pp. 411 - 423, 2021. DOI: 10.13189/ujaf.2021.090316.

(b): Roslin Lazarus, Stanley Lazarus, Shobhna Gupta (2021). Influence on Corporate Performance by Determinants of Working Capital. Universal Journal of Accounting and Finance, 9(3), 411 - 423. DOI: 10.13189/ujaf.2021.090316.

Copyright $\mathrm{C} 2021$ by authors, all rights reserved. Authors agree that this article remains permanently open access under the terms of the Creative Commons Attribution License 4.0 International License

\begin{abstract}
Working capital (WC) determinants play a crucial role in operating activities and corporate performance in a routine firm. This paper examines the influence of WC determinants on corporate performance employing a sample of thirty-one metal and mining firms listed on the National Stock Exchange (NSE), India for 10 years from 2010-2019. Pearson's correlation and fixed effect regression models were used to estimate the explanatory variables regression coefficients on the outcome variables. ROTA, ROEQ and ROCE variables were used to ascertain performance of sample firms and results revealed a significant negative influence of $\mathrm{WC}-$ inventory period (INVP) and accounts receivable collection period) on corporate performance (ROTA,ROEQ \& ROCE). No significant influence was observed between Corporate performance (ROTA, ROEQ $\&$ ROCE) and account Payable (AP). Moreover, Control variables corporate size $(\mathrm{CZ})$ results revealed significant negative influence on corporate performance (ROTA,ROEQ \& ROCE), \& Debt ratio(DR) result revealed a negative significant influence on ROTA, ROEQ .The result of sales Growth (SG) indicated a positive significant influence of corporate performance (ROTA,ROEQ \& ROCE). From the result of this study, it has been inferred that by reducing the INVP \& ARCP the management can enhance corporate performance \& eventually creating value for firm's shareholders. Through a meticulous reduction of the inventory period (INVP) \& account receivable collection period (ARCP), firms can demonstrate the effective and efficient utilization of financial resources, mitigating default \& liquidity risk
\end{abstract}

thereby enhancing its profit. Thus, systematic utilization of working capital elements positively influences firm's performance.

Keywords Inventory Period (INVP), Accounts Receivable Collection Period (ARCP), Accounts Payable Period (APP), Return on Assets (ROTA), Return on Equity (ROEQ), Return on Capital employed (ROCE), Working Capital (WC), India

JEL Classification: G30, G32

\section{Introduction}

Working capital (WC) determinants and its efficient utilization are crucial for routine smooth functioning of firm's operating activities and in enhancing its performance. Satisfactory level of a firm's working capital $\&$ its efficient utilization ensures sound liquidity position of corporate to meet the requirements of its short-period obligation, to mitigate associated risk and at enhancing profitability of firms. A high level of investment in WC results in sound liquidity position of corporates and overcapitalization. The inefficient utilization will have adverse impact on corporate profitability whereas low investment in WC, resulting in low corporate liquidity position but the efficient utilization WC determinants result in improved profitability. As the utilization of Working capital (WC) influence corporate performance it 
is crucial to ascertain the critical determinants of $\mathrm{WC}$ and examine its influence on corporate performance.

The present study investigates the influence of various determinants of Working capital (WC) on performance of metal and mining firms listed on the National Stock Exchange, India.

\section{Literature Review}

Working capital and firms' performance has been studied with diverse viewpoints and in different economic environments by various authors. Literature review considered in this study are as follows.

Sharma and Kumar [1] use OLS multiple regression to collect data during 2000 to 2008 from 263 non-financial listed at the BSE India examined the effect of working capital on profitability of Indian firms. Results disclosed that inventory days (INV) and accounts payable (AP) are negatively correlated with a firm's profitability, though, accounts receivables days (AR) and cash conversion period(CCC) found a positive relationship with the profitability of Indian companies. A comprehensive measure of working capital (cash conversion cycle) and profitability showed a positive association as against the theoretical background.

Makori and Jagongo [6] examined the effect of working capital management on firm's profitability employing balanced panel data of five manufacturing and construction firms, Kenya for a period of 2003 to 2012 from listed on the Nairobi Securities Exchange (NSE). Results showed a negative correlation existed between Return on Assets and the firms accounts receivable \& cash conversion cycle. Moreover, the study suggests a positive association between Inventory Turnover and also a positive association between Accounts payment period and Return on Assets. The study recommends a longer credit period for the firms to realize higher profitability. There exists positive association between Inventory Turnover in Days and Return on Assets, which implies that firms, which maintain sufficiently high inventory levels, reduce costs of possible interruptions in the production process and loss of business due to scarcity of products. The study recommends that the longer the accounts payable, the better the profitability.

Kajola, Nwaobia and Adedeji [2] a sample of 30 manufacturing firms listed on nigeran stock exchange for 7 years from 2004-2010 were employed to examine the impact of working capital management on firms' financial performance. The study revealed that average collection period \& working capital management (cash conversion cycle ) exerted a negative impact on profitability \& average payment period exerted significant positive relationship on firm's profitability. The study suggests that efforts should be directed to the optimum management of accounts receivables. Study result revealed a positive and significant relationship between average payable payment period and firm's profitability. Results implied positive affect on the profitability of the firm when the firm delays its payment to its creditors. Suggesting a caution to be employed because of the possibility of the suppliers blacklisting the firm and the extension of credit facility might be curtailed in the long run.

Salman, Folajin and Oriowo [4] investigated the relationship between working capital management on organizational profitability for manufacturing firms quoted in Nigerian Stock Exchange from 2005 -2013 comprising (20) manufacturing sectors. The study result revealed significant negative relationship between working capital $\&$ the return of assets and return on equity at $5 \%$ level \& suggested that firms' performance can be increased with short size of Cash Conversion Cycle.

Kasozi [5] examined the effect of working capital management on profitability using a sample of 69 manufacturing firms listed during the period 2007 to 2016 in South Africa.The results showed significant negative effects of average collection period and average payment period on profitability. In addition, Kasozi (2017) also observed a statistically significant positive relation existed between the average inventory days and profitability. The result implied that firms that proactively managed their trade receivables enhanced their profitability and firms that paid their trade payables on time performed better than those that delay such payments. Research results also revealed that manufacturing firms that stock up and maintain their inventory levels did not suffer from stock-outs and/or face challenges of securing finance to invest in such inventory resulting in operational efficiency and the enhancement of firm profitability in the long run.

Chowdhury, Alam, Sultana, and Hamid [3] examined the effects of working capital management (WCM) on the profitability of pharmaceutical companies listed on Dhaka Stock Exchange (DSE), in Bangladesh during the period of 2001-15. In regression analysis revealed a significant negative relationship between ROA and ICP, ACP \&CCC; return of equity and APP; EPS and ACP and APP whereas significant positive relationships are seen between APP and ROA; and CCC and ROE.As the maximum number $(80 \%)$ of variables has shown significant relationships with ROA which is a more stable variable comparing to ROE and EPS. It is concluded that through the efficient management of working capital by exploiting the relationships between profitability and working capital variables firms financial managers will create value for their shareholders and firms need to dedicate considerable amount of resources in developing effective working capital management policy.

Precisely, all the above research revealed that corporate profitability has been influenced by the determinants of Working capital (WC).

Research Gap- Although, numerous research work 
been carried out with regard to working capital (WC) and corporate performance but, only very few studies have been done on metal and mining sector. Metal and mining sector of India contributes significantly to the economy of India as it plays a crucial role in the survival and growth of almost every important sector like, construction, infrastructure development, automotive manufacturing, transportation, power generation. With the strong current and expected growth even in the residential and commercial building industry the demand for iron and steel is set to continue. [7]India was the third largest producer of coal and its production in financial year 20192020 stood at 729.10 million tons and reached 361.46 million tons in financial year 2020 -21 (as of November 2020).India ranks fourth in terms of iron ore production worldwide, and the production of iron ore stood at 206.45 MT in financial year 2019-2020. India has $8 \%$ of the world's iron ore deposits. India became the world's second largest crude steel producer in 2019 with production at 111.2 MT. Production of aluminium stood at 3.65 MT in Financial year 2019-2020 and in financial year 2020-21 (till January 2020), aluminium export from the country reached US\$ 18.24 million. Considering the significant contribution of metal and mining sector of India in development of the economy of India, the present paper aims to investigates the influence Working capital (WC) some key determinants on performance of metal and mining firms listed on the National Stock Exchange (NSE). Over the last ten years, financial data have been collected for this study \& influence on performance was examined using three outcome variables ROTA, ROEQ and ROCE which try to fill the research gap and so, the study is an attempt to contribute to the prevailing literature developed by previous authors.

\subsection{Research Aims}

The prime aim of this paper is to explore the influence of determinants of working capital (WC) on performance of Indian metal and mining firms listed on the National Stock Exchange (NSE). The following specific aims were structure to achieve the prime objective:

- To explore the predictor and outcome variables of Working capital (WC) and Corporate performance.
To examine the existence of correlation in determinants of working capital(WC) and performance (as measured by ROTA, ROEQ \& ROCE).

- To investigate the influence of unit change in predictor variables of working capital(WC) on predicted variables of performance as ascertained by ROTA, ROEQ \& ROCE on listed Indian metal and mining firms.

\section{Research Methodology}

\subsection{Research Sample and Financial Data Cumulation}

Since the working capital requirement vary from sectors and industry to industry requirements, some sectors require more working capital than others and firms operating in different sectors have different choices of the short-term investment and financing options. The selection of sample firms was based on following criterion. First, metal and mining firms and must be listed on National Stock Exchange, India before April 2008. Second, the stocks of the selected firms were listed during the duration of the study period. Third, firms having incomplete data of required variables for study period were also eliminated from the final sample considered for this paper. Finally, a sample of thirty-one metal and mining firms listed on National Stock Exchange (NSE),India for financial years from 2009-2010 to 2018-2019 (10 years) with thirty-one firm-year observations. Relevant research data were gathered from the audited financial statement data from firm's annual reports and stockaxis.com database holding financial information on NSE listed firms.

The data was analyzed using EViews. Pearson's Correlation Coefficient was calculated to examine correlation between various determinants of working capital and performance. Panel data regression analysis fixed effect models were employed to investigate the influence of unit change in predictor variables on predicted variables and to test the hypotheses. 


\subsection{Research Variables}

Table 1. outlines the variables that were used in this, along with their abbreviations and description.

\begin{tabular}{|c|c|c|}
\hline Variables/Acronyms & Type & Description \\
\hline Return on Assets (ROTA) & $\begin{array}{c}\text { Outcome } \\
\text { variable }\end{array}$ & $\begin{array}{l}\text { Measures how efficiently firm's management has generated earnings by utilizing its } \\
\text { total assets. }\end{array}$ \\
\hline Return on Equity (ROEQ) & $\begin{array}{l}\text { Outcome } \\
\text { variable }\end{array}$ & Measures how profitably a firm employs the total amount of shareholder equity. \\
\hline $\begin{array}{l}\text { Return on Capital } \\
\text { Employed (ROCE) }\end{array}$ & $\begin{array}{l}\text { Outcome } \\
\text { variable }\end{array}$ & $\begin{array}{l}\text { Ratio which measures how efficiently \& profitably a firm employs the available } \\
\text { funds(Firm's equity plus Non-current liabilities). }\end{array}$ \\
\hline Inventory period (INVP) & $\begin{array}{l}\text { Explanatory } \\
\text { variable }\end{array}$ & $\begin{array}{l}\text { Indicates the average number of days a firm holds on to their inventory before it is } \\
\text { sold. Lower the number of days the shorter funds tied in inventory. }\end{array}$ \\
\hline $\begin{array}{c}\text { Accounts Receivable } \\
\text { Collection Period(ARCP) }\end{array}$ & $\begin{array}{l}\text { Explanatory } \\
\text { variable }\end{array}$ & Indicates the average time taken by a firm to collect cash from its accounts receivables. \\
\hline $\begin{array}{l}\text { Accounts Payable Period } \\
\text { (APP) }\end{array}$ & $\begin{array}{l}\text { Explanatory } \\
\text { variable }\end{array}$ & Indicates average time taken by a firm to pay its accounts payable. \\
\hline Current Ratio (CR) & Control variable & $\begin{array}{l}\text { Measures the ability of firms to meet its short-term obligations due during the financial } \\
\text { year also the overall health of a firm. }\end{array}$ \\
\hline Corporate $\operatorname{Size}(\mathrm{CZ})$ & Control variable & Corporate Size as measured by natural logarithm of firm's total assets. \\
\hline Debt ratio $(\mathrm{DR})$ & Control variable & The ratio indicates the portion of firm's total assets that are financed by its total debts. \\
\hline Sales Growth (SG) & Control variable & $\begin{array}{c}\text { It measures the growth of sales in the current year as compare to previous financial } \\
\text { years. }\end{array}$ \\
\hline
\end{tabular}

\subsection{Research Hypotheses}

Based on a study of research literature \& results from previous researchers the following hypotheses were developed to accomplish the study aims.

\section{Hypothesis 1}

$\mathrm{H}_{0}$ : INVP exerts no significant influence on ROTA, ROEQ \& ROCE).

$\mathrm{H}_{1}$ : INVP exerts a Significant influence on ROTA, ROEQ \& ROCE).

\section{Hypothesis 2}

H0: ARCP exerts no significant influence on ROTA, ROEQ \& ROCE).

H1: ARCP exerts a Significant influence on ROTA, ROEQ \& ROCE).

\section{Hypothesis 3}

H0: APP exerts no significant influence on ROTA, ROEQ \& ROCE).

H1: APP exerts a Significant influence on ROTA, ROEQ \& ROCE).

\subsection{Conceptual Framework}

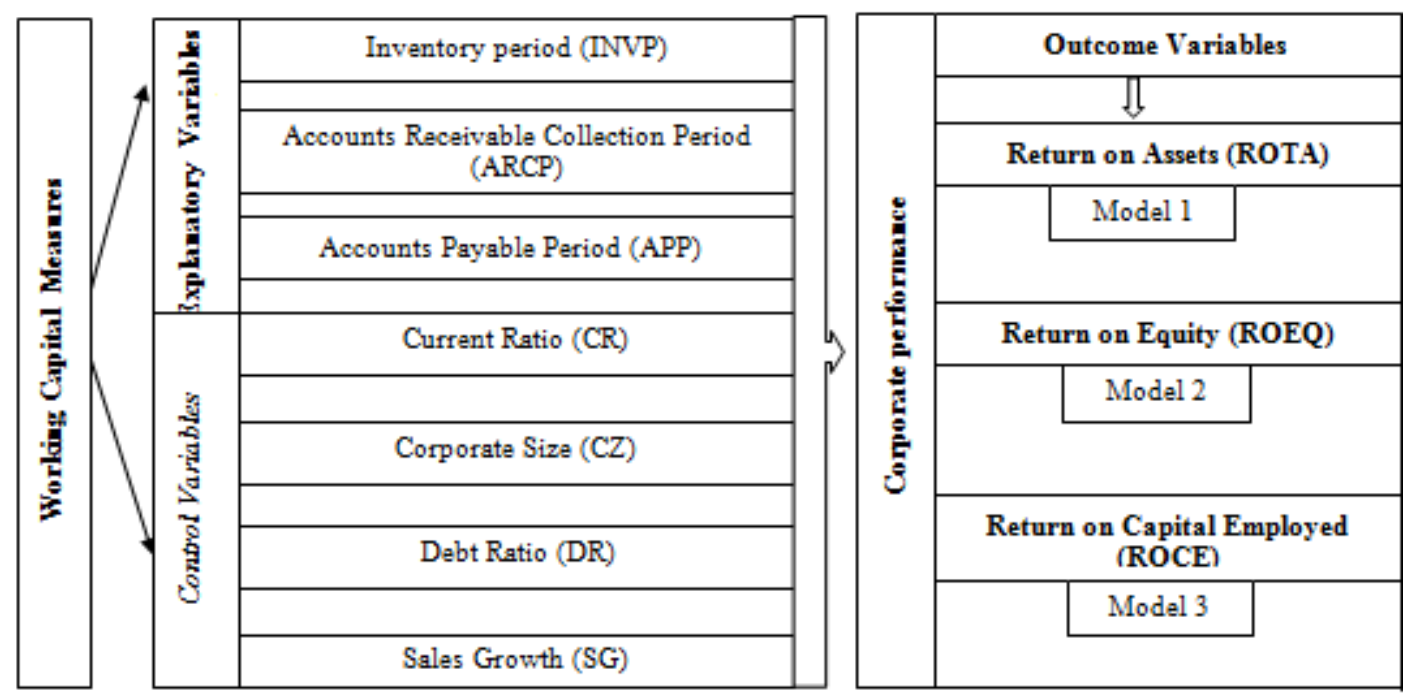

Figure 1. Conceptual framework underlying the proposed influence on corporate performance by determinants of working capital (WC) 


\subsection{Regression Models}

Regression models were structured to investigate the influence of determinants of WC on corporate performance. Each explanatory variable along with the control variables were regressed against the three outcome variables. Three equations were structured in each model.

Model 1: examines the influence of INVP \& control variables on performance as ascertain by ROTA, ROEQ \&ROCE.

Equation 1

$$
\begin{aligned}
& \text { ROTA }_{\text {it }}=\beta 0+\beta 1 \text { INVPit }+\beta_{2} \mathrm{CR}_{\text {it }}+\beta_{3} \mathrm{CZ}_{\text {it }}+\beta_{4} \mathrm{DR}_{\text {it }}+ \\
& \beta_{5} \mathrm{SG}_{\text {it }}+\varepsilon_{\text {it. }}
\end{aligned}
$$

Equation 2

$$
\begin{gathered}
\operatorname{ROEQ}_{i t}=\beta 0+\beta 1 \mathrm{INVPit}+\beta_{2} \mathrm{CR}_{\text {it }}+\beta_{3} \mathrm{CZ}_{\text {it }}+\beta_{4} \mathrm{DR}_{\text {it }}+ \\
\beta_{5} \mathrm{SG}_{\mathrm{it}}+\varepsilon_{\text {it. }}
\end{gathered}
$$

Equation 3

$$
\begin{gathered}
\operatorname{ROCE}_{\text {it }}=\beta 0+\beta 1 \mathrm{INVPit}+\beta_{2} \mathrm{CR}_{\text {it }}+\beta_{3} \mathrm{CZ}_{\text {it }}+\beta_{4} \mathrm{DR}_{\text {it }}+ \\
\beta_{5} \mathrm{SG}_{\text {it }}+\varepsilon_{\text {it. }}
\end{gathered}
$$

Model 2: examines the influence of ARCP \& control variables on performance as ascertain by ROTA, ROEQ \& ROCE.

Equation 1

$$
\begin{gathered}
\operatorname{ROTA}_{\text {it }}=\beta 0+\beta_{1} \mathrm{ARCP}_{\text {it }}+\beta_{2} \mathrm{CR}_{\text {it }}+\beta_{3} \mathrm{CZ}_{\text {it }}+\beta_{4} \mathrm{DR}_{\text {it }}+ \\
\beta_{5} \mathrm{SG}_{\text {it }}+\varepsilon_{\text {it. }}
\end{gathered}
$$

Equation 2

$$
\begin{gathered}
\operatorname{ROEQ}_{i t}=\beta 0+\beta_{1} \mathrm{ARCP}_{\text {it }}+\beta_{2} \mathrm{CR}_{\text {it }}+\beta_{3} \mathrm{CZ}_{\text {it }}+\beta_{4} \mathrm{DR}_{\text {it }}+ \\
\beta_{5} \mathrm{SG}_{\text {it }}+\varepsilon_{\text {it. }}
\end{gathered}
$$

Equation 3

$$
\begin{gathered}
\operatorname{ROCE}_{\text {it }}=\beta 0+\beta_{1} \mathrm{ARCP}_{\text {it }}+\beta_{2} \mathrm{CR}_{\text {it }}+\beta_{3} \mathrm{CZ}_{\text {it }}+\beta_{4} \mathrm{DR}_{\text {it }}+ \\
\beta_{5} \mathrm{SG}_{\mathrm{it}}+\varepsilon_{\text {it. }}
\end{gathered}
$$

Model 3: examines the influence of APP \& control variables on performance as ascertain by ROTA, ROEQ \& ROCE.

Equation 1

$$
\begin{array}{r}
\mathrm{ROTA}_{\text {it }}=\beta 0+\beta_{1} \mathrm{APP}_{\text {it }}+\underset{\text { it }}{\beta_{2} \mathrm{CR}_{\text {it }}+\varepsilon_{\text {it. }}}+\beta_{3} \mathrm{CZ}_{\text {it }}+\beta_{4} \mathrm{DR}_{\text {it }}+\beta_{5} \mathrm{SG} \\
\text { Equ }
\end{array}
$$

Equation 2

$$
\begin{gathered}
\mathrm{ROEQ}_{\mathrm{it}}=\beta 0+\beta_{1} \mathrm{APP}_{\mathrm{it}}+\beta_{2} \mathrm{CR}_{\text {it }}+\beta_{3} \mathrm{CZ}_{\text {it }}+\beta_{4} \mathrm{DR}_{\text {it }}+\beta_{5} \mathrm{SG} \\
\text { it }+\varepsilon_{\text {it. }}
\end{gathered}
$$

Equation 3

$$
\begin{aligned}
& \mathrm{ROCE}_{\text {it }}=\beta 0+\beta_{1} \mathrm{APP}_{\mathrm{it}}+\beta_{2} \mathrm{CR}_{\mathrm{it}}+\beta_{3} \mathrm{CZ}_{\mathrm{it}}+\beta_{4} \mathrm{DR}_{\mathrm{it}}+\beta_{5} \mathrm{SG} \\
& \text { it }+\varepsilon_{\text {it. }}
\end{aligned}
$$

Model 4: examines the influence of working capital all Outcome variables \& control variables on performance as ascertain by ROTA, ROEQ \&ROCE.

Equation 1

ROTA $_{\text {it }}=\beta 0+\beta_{1} \mathrm{INVPit}+\beta_{2} \mathrm{ARCP}_{\text {it }}+\beta_{3} \mathrm{APP}_{\text {it }}+\beta_{4} \mathrm{CR}_{\text {it }}+$ $\beta_{5} \mathrm{CZ}_{\text {it }}+\beta_{6} \mathrm{DR}_{\text {it }}+\beta_{7} \mathrm{SG}_{\text {it }}+\varepsilon_{\text {it. }}$

Equation 2 ROEQ $_{\text {it }}=\beta 0+\beta_{1}$ INVPit $+\beta 2 A R C P i t+\beta 3$ APPit $+\beta 4 C R$
it $+\beta 5$ CZ it $+\beta 6$ DR it $+\beta 7$ SG it $+\varepsilon_{\text {it. }}$

Equation 3

ROCE $_{\text {it }}=\beta 0+\beta_{1} \mathrm{INVPit}+\beta_{2} \mathrm{ARCP}_{\mathrm{it}}+\beta_{3} \mathrm{APP}_{\mathrm{it}}+\beta_{4} \mathrm{CR}_{\mathrm{it}}+$ $\beta_{5} \mathrm{CZ}_{\text {it }}+\beta_{6} \mathrm{DR}_{\text {it }}+\beta_{7} \mathrm{SG}_{\text {it }}+\varepsilon_{\text {it. }}$

\section{Empirical Analysis and Findings of the Study}

This section presents the empirical analysis of quantitative data using E-Views 8. Firstly, descriptive analysis is addressed followed by correlation \& regression analysis.

\section{4.(a) Descriptive Statistics}

Table 2 address the descriptive statistics of the collected variables thirty-one metal and mining sector firms listed on NSE, India for financial years from 2009-2010 to 2018-2019 with 310 firm-year observations. The mean, median, standard deviation, the minimum and maximum values and the research variables of the study period are presented in descriptive statistics.

Descriptive statistics as depicted in table 2, the average value of for 310 firm year observations firms ROTA was $6 \%$, ROEQ was $11 \%$ \& ROCE was $16 \%$ with a respective median of $5 \%, 10 \%$ and $13 \%$. It deviated by $6 \%, 16 \%$ and $13 \%$ to the left and right side of the respective mean value. The average inventory conversion period to transform their inventories to sales revenue was 61 days, the credit period allowed by sampled firms to their receivables ranged on an average at 38 days \& account payable period on average the sampled firms took on an average was 35 days to clear dues of its trade payables for credit purchases with a respective standard dev of 36 days for INVP, 24 days for ARCP \& 20 days for APP.

In addition, control variables descriptive statistics results of selected Indian metal \& mining firms revealed an average current ratio of 2.31 indicating a sound liquidity management of most of the sample firms. The average Corporate Size 7.96, Debt ratio of $51 \%$ and sales growth was $12 \%$ with a respective standard deviation of $2.37,2.090 .20 \%$ and $23 \%$. 
Table 2. Descriptive statistics of 310 firm year observation

\begin{tabular}{|c|c|c|c|c|c|c|c|c|c|c|}
\hline & ROTA & ROEQ & ROCE & INVP & $\mathrm{ARCP}$ & APP & $\mathrm{CR}$ & $\mathrm{CZ}$ & DR & SG \\
\hline Mean & 0.06 & 0.11 & 0.16 & 61 & 38 & 35 & 2.31 & 7.96 & 0.51 & 0.12 \\
\hline Median (M) & 0.05 & 0.10 & 0.13 & 56 & 36 & 31 & 1.49 & 7.88 & 0.56 & 0.09 \\
\hline Maximum (Max) & 0.28 & 1.16 & 1.09 & 185 & 131 & 122 & 13.60 & 12.36 & 0.93 & 0.90 \\
\hline Minimum (Min) & -0.10 & -0.87 & -0.06 & 6 & 2 & 0 & 0.55 & 3.66 & 0.07 & -0.48 \\
\hline Std. Dev. $(\sigma)$ & 0.06 & 0.16 & 0.13 & 36 & 24 & 21 & 2.37 & 2.09 & 0.20 & 0.23 \\
\hline Skewness & 0.85 & 0.17 & 2.18 & 0.95 & 1.10 & 1.13 & 2.95 & 0.27 & -0.54 & 0.58 \\
\hline Kurtosis & 3.79 & 14.61 & 11.73 & 3.60 & 4.43 & 4.93 & 11.64 & 2.42 & 2.33 & 3.98 \\
\hline Jarque-Bera & 45.78 & 1743.04 & 1230.42 & 51.17 & 88.43 & 114.56 & 1414.29 & 8.14 & 21.04 & 29.63 \\
\hline Probability & 0.00 & 0.00 & 0.00 & 0.00 & 0.00 & 0.00 & 0.00 & 0.02 & 0.00 & 0.00 \\
\hline Sum & 18.98 & 35.59 & 50.77 & 18867.00 & 11776.00 & 10853.00 & 716.78 & 2466.10 & 158.68 & 35.98 \\
\hline Sum Sq. Dev. & 1.27 & 7.77 & 5.25 & 393871.00 & 181249.90 & 128171.00 & 1741.44 & 1355.83 & 12.72 & 15.75 \\
\hline Observations & 310 & 310 & 310 & 310 & 310 & 310 & 310 & 310 & 310 & 310 \\
\hline
\end{tabular}

Table 3. Pearson's correlation analysis for Indian metal \& mining companies, 310 firms - year observations

\begin{tabular}{|c|c|c|c|c|c|c|c|c|c|c|}
\hline \multicolumn{11}{|c|}{ Correlation } \\
\hline & ROTA & ROEQ & ROCE & INVP & $\mathrm{ARCP}$ & APP & $\mathrm{CR}$ & $\mathrm{CZ}$ & DR & SG \\
\hline ROTA & 1.000 & & & & & & & & & \\
\hline ROEQ & $0.778 * * *$ & 1.000 & & & & & & & & \\
\hline ROCE & $0.816^{* * * *}$ & $0.784 * * *$ & 1.000 & & & & & & & \\
\hline INVP & $-0.469 * * *$ & $-0.389 * * *$ & $-0.429 * * *$ & 1.000 & & & & & & \\
\hline $\mathrm{ARCP}$ & $-0.262 * * *$ & $-0.250 * * *$ & $-0.170 * * *$ & $0.393 * * *$ & 1.000 & & & & & \\
\hline APP & $-0.271 * * *$ & $-0.194 * * *$ & $-0.191 * * *$ & $0.319 * * *$ & $0.148 * * *$ & 1.000 & & & & \\
\hline $\mathrm{CR}$ & $0.457 * * *$ & $0.167 * * *$ & $0.214 * * *$ & $-0.134 * *$ & 0.033 & $-0.207 * * *$ & 1.000 & & & \\
\hline $\mathrm{CZ}$ & $0.131 * *$ & 0.058 & $0.167 * * *$ & $0.155^{* * *}$ & $-0.180 * * *$ & $0.240 * * *$ & $0.120^{* *}$ & 1.000 & & \\
\hline DR & $-0.575 * * *$ & $-0.201 * * *$ & $-0.217 * * *$ & $0.277 * * *$ & $0.181 * * *$ & $0.321 * * *$ & $-0.685^{* * *}$ & -0.020 & 1.000 & \\
\hline SG & $0.177 * * *$ & $0.223 * * *$ & $0.167 * * *$ & $-0.179 * * *$ & $-0.099^{*}$ & $-0.150 * * *$ & -0.080 & -0.162 & 0.038 & 1.000 \\
\hline
\end{tabular}

Note: $(* * *),(* *) \&(*)$ represent statistical significance at the 1,5 and 10 percent levels. 


\section{4.(a) Descriptive Statistics}

Table 3 reveals that Pearson correlation coefficients for most of the variables and coefficient measures for most measures are significant. Pearson correlation coefficients results revealed a negative and significant association between the measures of performance (ROTA, ROEQ, ROCE) and the working capital independent determinants Inventory period (INVP), Accounts Receivable Collection Period(ARCP), Accounts Payable(APP). This implys that the increase in firm's performance is related to lower inventory period (INVP), shorter accounts receivable collection period(ARCP), and shorter accounts payable period (APP). The result indicates that the increase in firm's performance is significantly associated with the firms holding the inventories for lesser number of days before selling \& collecting its revenue in shorter period from receivables and contrariwise. Also, increase in firm's performance is significantly associated with a shorter payable period probably firms seeking to take benefit of early payment discounts or working at improving its credit rating. Further, Current ratio (CR), Corporate size (CZ) \& sales growth (SG) were positively related to firms performance indicating that current ratio is increased, and thatbigger firms and higher sales growth are reported higher firms performance and decrease in firms performance with a firms with low current ratio, smaller firms and low sales growth. The correlation coefficients of the Debt ratio registered a negative \& significant association with firm's performance, which infers an increase in total debts of firm result in an decrease in firm's performance due to high financial cost.

Table 4.1. Multivariate regression estimates using fixed effect model for model 1 to investigate the influence of INVP \& control variables on performance as measured by ROTA, ROEQ \& ROCE.

\begin{tabular}{|c|c|c|c|c|c|c|}
\hline \multirow{2}{*}{$\begin{array}{c}\text { FE-Model } 1 \\
\text { Variable }\end{array}$} & \multicolumn{2}{|c|}{ Eq 1 -ROTA } & \multicolumn{2}{|c|}{ Eq 2-ROEQ } & \multicolumn{2}{|c|}{ Eq 3-ROCE } \\
\hline & Coefficient $(\beta)$ & (P-value) & Coefficient $(\beta)$ & (P-value) & Coefficient $(\beta)$ & (P-value) \\
\hline INVP & -0.000939 & 0.0000 & -0.001968 & 0.0002 & -0.001865 & 0.0000 \\
\hline $\mathrm{CR}$ & -0.000464 & 0.8139 & -0.000464 & 0.8139 & -0.003927 & 0.3488 \\
\hline $\mathrm{CZ}$ & -0.028676 & 0.0004 & -0.028676 & 0.0004 & -0.051839 & 0.0023 \\
\hline DR & -0.109127 & 0.0002 & -0.109127 & 0.0002 & -0.017221 & 0.7821 \\
\hline SG & 0.042078 & 0.0000 & 0.042078 & 0.0000 & 0.080823 & 0.0001 \\
\hline $\mathrm{C}$ & 0.398525 & 0.0000 & 0.398525 & 0.0000 & 0.698199 & 0.0000 \\
\hline R-squared value & & 0.725805 & & 0.445369 & & 0.700439 \\
\hline Adj R-squared value & & 0.690780 & & 0.374521 & & 0.662173 \\
\hline Log likelihood & & 612.4982 & & 222.9639 & & 378.9708 \\
\hline F-statistic & & 20.72255 & & 6.286336 & & 18.30487 \\
\hline Prob & & 0.000000 & & 0.000000 & & 0.000000 \\
\hline Durbin-Watson stat & & 1.163698 & & 1.557032 & & 1.276399 \\
\hline \multicolumn{7}{|l|}{ Hausman-Test Summary } \\
\hline Chi-Sq. Statistic & & 24.436810 & & 14.698132 & & 16.311124 \\
\hline Chi-Sq. d.f. & & 5 & & 5 & & 5 \\
\hline Prob. & & 0.0002 & & 0.0117 & & 0.0060 \\
\hline \multicolumn{7}{|l|}{ Wald Test: } \\
\hline \multicolumn{7}{|l|}{$F$-statistic } \\
\hline Value & & 30.80021 & & 20.04878 & & 16.86614 \\
\hline $\mathrm{df}$ & & $(5,304)$ & & $(5,274)$ & & $(5,274)$ \\
\hline Probability & & 0.0000 & & 0.0000 & & 0.0000 \\
\hline \multicolumn{7}{|l|}{ Chi-square } \\
\hline Value & & 154.0011 & & 100.2439 & & 84.33070 \\
\hline $\mathrm{df}$ & & 5 & & 5 & & 5 \\
\hline Probability & & 0.0000 & & 0.0000 & & 0.0000 \\
\hline
\end{tabular}

Research Periods - 10 years, thirty-one firms financial data \& total panel balanced observations: 310 


\section{4.(b) Panel data regression analysis}

Balanced panel data regression analysis was applied to estimate the influence working capital determinants have on the performance variables.

Hausman test was performed to establish appropriate model the fixed or random effects model.

$H_{0}:$ Random effects model (REM)

$H_{1}$ : Fixed effects model (FEM)

The result revealed that the probability value was less than $5 \%$ thereby alternative hypothesis is accepted and inferred that the Fixed effects model is appropriate. Moreover, to determine the accurate model between the Fixed effect model and the Pooled OLS regression model, the Wald test was applied.

Hypothesis: (HO) Pooled OLS regression model. (H1):Fixed effect model.

As the probability value of the Wald test is below $5 \%$ in all the cases, thereby accepting alternative hypothesis and considering the Fixed effects model (FEM) as appropriate.

Table 4.2. Multivariate regression estimates using fixed effect model for model 2 to investigate ACRP \& control variables influence of performance as measured by ROTA, ROEQ \& ROCE.

\begin{tabular}{|c|c|c|c|c|c|c|}
\hline \multirow{2}{*}{$\begin{array}{c}\text { FE-Model } 2 \\
\text { Variable }\end{array}$} & \multicolumn{2}{|c|}{ Eq 1 -ROTA } & \multicolumn{2}{|c|}{ Eq 2-ROEQ } & \multicolumn{2}{|c|}{ Eq 3-ROCE } \\
\hline & Coefficient( $(\beta)$ & (P-value) & Coefficient( $(\beta)$ & (P-value) & Coefficient( $(\beta)$ & (P-value) \\
\hline ARCP & -0.001013 & 0.0000 & -0.003636 & 0.0000 & -0.002330 & 0.0000 \\
\hline $\mathrm{CR}$ & 0.000156 & 0.9394 & 0.005274 & 0.4425 & -0.002348 & 0.5837 \\
\hline $\mathrm{CZ}$ & -0.030426 & 0.0003 & -0.083883 & 0.0025 & -0.053050 & 0.0022 \\
\hline DR & -0.137477 & 0.0000 & -0.051253 & 0.6139 & -0.077948 & 0.2196 \\
\hline SG & 0.046725 & 0.0000 & 0.123362 & 0.0003 & 0.086226 & 0.0001 \\
\hline $\mathrm{C}$ & 0.406344 & 0.0000 & 0.919964 & 0.0000 & 0.709632 & 0.0000 \\
\hline R-squared value & & 0.708198 & & 0.462736 & & 0.690615 \\
\hline Adj R-squared value & & 0.670924 & & 0.394107 & & 0.651095 \\
\hline Log likelihood & & 602.8514 & & 227.8950 & & 373.9694 \\
\hline F-statistic & & 18.99978 & & 6.742598 & & 17.47508 \\
\hline Prob & & 0.000000 & & 0.000000 & & 0.000000 \\
\hline Durbin-Watson stat & & 1.184682 & & 1.646915 & & 1.258309 \\
\hline \multicolumn{7}{|l|}{ Hausman-Test Summary } \\
\hline Chi-Sq. Statistic & & 23.993349 & & 19.204436 & & 17.856429 \\
\hline Chi-Sq. d.f. & & 5 & & 5 & & 5 \\
\hline Prob. & & 0.0002 & & 0.0018 & & 0.0031 \\
\hline \multicolumn{7}{|l|}{ Wald Test: } \\
\hline \multicolumn{7}{|l|}{$F$-statistic } \\
\hline Value & & 20.04878 & & 12.96259 & & 14.59060 \\
\hline $\mathrm{df}$ & & $(5,274)$ & & $(5,274)$ & & $(5,274)$ \\
\hline Probability & & 0.0000 & & 0.0000 & & 0.0000 \\
\hline \multicolumn{7}{|l|}{ Chi-square } \\
\hline Value & & 100.2439 & & 64.81297 & & 72.95300 \\
\hline $\mathrm{df}$ & & 5 & & 5 & & 5 \\
\hline Probability & & 0.0000 & & 0.0000 & & 0.0000 \\
\hline
\end{tabular}

Research Periods - 10 years, thirty-one firms financial data \& total panel balanced observations: 310. 
Table 4.3. Multivariate regression estimates using fixed effect model for model 3 to investigate APP \& control variables influence on performance as measured by ROTA, ROEQ \& ROCE.

\begin{tabular}{|c|c|c|c|c|c|c|}
\hline \multirow{2}{*}{$\begin{array}{c}\text { FE-Model } 3 \\
\text { Variable }\end{array}$} & \multicolumn{2}{|c|}{ Eq 1 -ROTA } & \multicolumn{2}{|c|}{ Eq 2-ROEQ } & \multicolumn{2}{|c|}{ Eq 3-ROCE } \\
\hline & Coefficient $(\beta)$ & (P-value) & Coefficient $(\beta)$ & (P-value) & Coefficient $(\beta)$ & (P-value) \\
\hline APP & -0.000220 & 0.1879 & -0.000403 & 0.4743 & $2.25 \mathrm{E}-07$ & 0.9995 \\
\hline $\mathrm{CR}$ & -0.001079 & 0.6077 & 0.001060 & 0.8812 & -0.004902 & 0.2705 \\
\hline $\mathrm{CZ}$ & -0.037348 & 0.0000 & -0.109298 & 0.0001 & -0.069718 & 0.0001 \\
\hline DR & -0.116040 & 0.0003 & 0.012699 & 0.9054 & -0.045661 & 0.4950 \\
\hline SG & 0.057146 & 0.0000 & 0.163982 & 0.0000 & 0.114409 & 0.0000 \\
\hline $\mathrm{C}$ & -0.000220 & 0.1879 & -0.000403 & 0.4743 & & \\
\hline R-squared value & & 0.688572 & & 0.418215 & & 0.662939 \\
\hline Adj R-squared value & & 0.648791 & & 0.343900 & & 0.619884 \\
\hline Log likelihood & & 592.7622 & & 215.5554 & & 360.6896 \\
\hline F-statistic & & 17.30909 & & 5.627561 & & 15.39743 \\
\hline Prob & & 0.000000 & & 0.000000 & & 0.000000 \\
\hline Durbin-Watson stat & & 1.096641 & & 1.547727 & & 1.170924 \\
\hline \multicolumn{7}{|c|}{ Hausman-Test Summary } \\
\hline Chi-Sq. Statistic & & 26.726162 & & 17.856429 & & 18.835532 \\
\hline Chi-Sq. d.f. & & 5 & & 5 & & 5 \\
\hline Prob. & & 0.0001 & & 0.0031 & & 0.0021 \\
\hline \multicolumn{7}{|l|}{ Wald Test: } \\
\hline \multicolumn{7}{|l|}{$F$-statistic } \\
\hline Value & & 17.74317 & & 14.59060 & & 7.777163 \\
\hline $\mathrm{df}$ & & $(5,304)$ & & $(5,274)$ & & $(5,274)$ \\
\hline Probability & & 0.0000 & & 0.0000 & & 0.0000 \\
\hline \multicolumn{7}{|l|}{ Chi-square } \\
\hline Value & & 88.71585 & & 72.95300 & & 38.88582 \\
\hline $\mathrm{df}$ & & 5 & & 5 & & 5 \\
\hline Probability & & 0.0000 & & 0.0000 & & 0.0000 \\
\hline
\end{tabular}

Research Periods - 10 years, thirty-one firms financial data \& total panel balanced observations: 310.

\section{4.(b) Panel data regression analysis}

Table $4.1,4.2 \& 4.3$ presents the regression result of Models 1, 2\& 3 with three Equations in each Model with dependent variable equation 1 - ROTA, equation 2 ROEQ and equation 3 -ROCE \& the independent variable for model 1 - inventory period (INVP),Model 2-accounts receivable collection period (ARCP) \& Model 3- accounts payable period (APP) \& finally, control variables as CR, CZ, DR \& SG in all three models . Model 1 - result divulged a statistically significant negative influence of INVP on ROTA, ROEQ \& ROCE, inferring that shorter inventory days improves firm's performance and contrariwise. Results revealed that the firms that turned its inventory into sales in lesser days had a positive impact on corporate performance because of income generation, the decrease in holding costs, and the mitigating risk of inventory obsolescence. The lower the inventory turnover days the faster corporate management can release the cash tied in inventory and use it for the replenishment of inventory \& sufficient cash for meeting short term obligations and to generate good earnings. Eventually resulting at utilizing its total assets efficiently \& firm will be able to effectively employ the total amount of shareholder's equity\& also the total available funds(Firm's equity plus Non-current liabilities) to generate good return. This result is analogous with the earlier research work of Sharma and Kumar[1] \& Chowdhury, Alam, Sultana, and Hamid[3] where ROA was a dependent variable to measure performance. However, this is contrary to the result of Makori and Jagongo [6] \& Kasozi[5] where inventory days and performance revealed a significant positive relationship.

Model 2 revealed a statistically significant negative influence of ARCP on ROTA, ROEQ \& ROCE, inferring that the shorter accounts receivable collection period, 
(ARCP) the higher the firm's performance and contrariwise. Result revealed that the lower cash collection receivables period reaps higher corporate performance, the less the credit period and efficient collection of cash from receivables and the more efficiently a firm's management can generate earnings by utilizing the available cash and other resources efficiently. Moreover, the result revealed that firms applying conservative credit policy had employed the amount invested by its shareholder's \& funds generated through non-current liabilities effectively\& efficiently thus mitigating the risk of default/irrecoverable by receivables and liquidity problems. This result as regards to ROTA is analogous to previous study results of Makori and Jagongo [6], Nwaobia and Adedeji [2] \& Kasozi [5] \& Chowdhury, Alam, Sultana, and Hamid [3] where ROA was a dependent variable to gauge performance. However, this is contrary to Sharma and Kumar's[1] study, which exhibited a positive relationship between accounts receivables days $\&$ corporate profitability.

Model 3 revealed a negative insignificant influence of APP on ROTA, ROEQ \& ROCE, inferring that firms paying payables promptly as having shorter payable period results in increase in firm's performance, probably because firms availed the benefit of early payment discounts impacting positively the profit of the firm, and by avoiding any penalties of delay payment, moreover in future such firms may be aimed at improving its credit rating with suppliers to get higher credit period in future and utilizing the available cash effectively for some other operational purpose.

Regarding control variables, the result revealed a significant negative influence of $\mathrm{CZ}$ on ROTA, ROEQ \& ROCE, revealing a smaller firm enjoying greater performance with regards to return on assets, return on equity and return on capital employed as they may require less working capital and can manage it efficiently thereby creating good returns. So, the smaller the corporate size, the higher will be the degree of efficient utilization of working capital resulting in increase in profit. Debt ratio(DR) result revealed a significant negative influence on ROTA, ROEQ in Model 1 and on ROTA in Model 2 \& 3. The lower the total debt to total asset, the higher the return on assets and Return on equity. Firms that relied less on debts (due to high finance cost and respective business's financial risk) were able to generate higher returns. Sales Growth (SG) result indicated a significant positive influence on ROTA, ROEQ \& ROCE in all the three Models (table 4.1, 4.2 \& 4.3). As evident from correlation \& regression result shorter INVP,ARCP\& APP period contributes an increase in sales and eventually resulting in the increase in corporate performance.So, firms that were able to generate higher sales revenue has generated increased corporate performance.

Model 1- adjusted R2 of Equation1 is $69.1 \%$ (F statistics of 20.72), Equation 2 is $37.4 \%$ (F statistics of 6.29), -Equation 3 is $66.2 \%$ (F statistics of 18.31) which infers that the changes in corporate performance (ROTA, ROEQ \& ROCE) were driven by the Working capital (WC) variables considered in this model. The adjusted R2 of ROTA and ROCE revealed strong fit and ROEQ moderate fit, all the three models are acceptable as $\mathrm{p}$-value $=.000$, indicating model 1 is well fit at 1 percent level of significance level.

Finally, the alternate hypothesis1 - $\mathrm{H} 1$ is accepted as negative significant association which exists between INVP and Corporate performance as measured by ROTA, ROEQ\& ROCE. Model 2- adjusted R2 of Equation1 is $67.1 \%$ (F statistics of 19),Equation 2 is $39.4 \%$ (F statistics of 6.74), -Equation 3 is $65.1 \% \%$ (F statistics of 17.47)which infers that the changes in corporate performance (ROTA, ROEQ \& ROCE) were driven by the Working capital (WC) variables considered in this model.

The adjusted R2 of ROTA and ROCE revealed strong fit and ROEQ moderate fit. Overall, all the three models are acceptable as p-value $=.000$, indicating that model 1 is well fit at 1 percent level of significance level.

Finally, the alternate hypothesis $2-\mathrm{H} 1$ is accepted as negative significant association, which exists between ARCP and Corporate performance as measured by ROTA, ROEQ \& ROCE.

Model 3- adjusted R2 Equation1 is $64.9 \%$ (F statistics of 17.31 ), Equation 2 is $34.4 \%$ ( $\mathrm{F}$ statistics of 5.63 ), -Equation 3 is $61.9 \%$ (F statistics of 15.4) which infers that the changes in corporate performance (ROTA, ROEQ \& ROCE) were driven by the Working capital (WC) variables considered in this model.

The adjusted R2 of ROTA and ROCE revealed strong fit and ROEQ moderate fit. Overall, all the three models are acceptable as $p$-value $=.000$, indicating model 1 is well fit at 1 percent level of significance level.

Finally, The Null hypothesis $3-\mathrm{H} 0$ is accepted no association exists between APP and Corporate performance as ascertained by ROTA, ROEQ \& ROCE. 
Table 4.4. Multivariate regression estimates using fixed effect model 4 for model 1 to investigate the influence of INVP, ARCP,APP \& control variables on performance as measured by ROTA, ROEQ\& ROCE.

\begin{tabular}{|c|c|c|c|c|c|c|}
\hline \multirow{2}{*}{$\begin{array}{c}\text { FE-Model } 4 \\
\text { Variable } \\
\end{array}$} & \multicolumn{2}{|c|}{ ROTA } & \multicolumn{2}{|c|}{ ROEQ } & \multicolumn{2}{|c|}{ ROCE } \\
\hline & Coefficient $(\beta)$ & P-value & Coefficient $(\beta)$ & P-value & Coefficient( $\beta)$ & P-value \\
\hline INVP & -0.000842 & 0.0000 & -0.001579 & 0.0026 & -0.001636 & 0.0000 \\
\hline $\mathrm{ARCP}$ & -0.000781 & 0.0004 & -0.003215 & 0.0000 & -0.001934 & 0.0000 \\
\hline APP & -0.000154 & 0.3147 & -0.000171 & 0.7492 & 0.000153 & 0.6368 \\
\hline $\mathrm{CR}$ & 0.000254 & 0.8961 & 0.005541 & 0.4144 & -0.001841 & 0.6540 \\
\hline $\mathrm{CZ}$ & -0.023786 & 0.0027 & -0.071505 & 0.0097 & -0.040428 & 0.0156 \\
\hline DR & -0.116224 & 0.0001 & -0.015583 & 0.8791 & -0.052669 & 0.3961 \\
\hline SG & 0.033083 & 0.0010 & 0.098594 & 0.0048 & 0.062835 & 0.0030 \\
\hline $\mathrm{C}$ & 0.391842 & 0.0000 & 0.891605 & 0.0001 & 0.676990 & 0.0000 \\
\hline R-squared value & & 0.739821 & & 0.480582 & & 0.718803 \\
\hline Adj R-squared value & & 0.704428 & & 0.409926 & & 0.680552 \\
\hline Log likelihood & & 620.6306 & & 233.1312 & & 388.7769 \\
\hline F-statistic & & 20.90358 & & 6.801713 & & 18.79173 \\
\hline Prob & & 0.000000 & & 0.000000 & & 0.000000 \\
\hline $\begin{array}{c}\text { Durbin-Watson stat } \\
\text { Hausman-Test } \\
\text { Summary }\end{array}$ & & 1.244161 & & 1.649723 & & 1.341859 \\
\hline Chi-Sq. Statistic & & 29.064390 & & 22.923156 & & 23.305821 \\
\hline Chi-Sq. d.f. & & 7 & & 7 & & 7 \\
\hline Prob. & & 0.0001 & & 0.0018 & & 0.0015 \\
\hline \multicolumn{7}{|l|}{ Wald Test: } \\
\hline \multicolumn{7}{|l|}{ F-statistic } \\
\hline Value & & 20.66664 & & 10.84232 & & 15.27807 \\
\hline $\mathrm{df}$ & & $(7,272)$ & & $(7,272)$ & & $(7,272)$ \\
\hline Probability & & 0.0000 & & 0.0000 & & 0.0000 \\
\hline \multicolumn{7}{|l|}{ Chi-square } \\
\hline Value & & 144.6665 & & 75.89626 & & 106.9465 \\
\hline $\mathrm{df}$ & & 7 & & 7 & & 7 \\
\hline Probability & & 0.0000 & & 0.0000 & & 0.0000 \\
\hline
\end{tabular}

Research Periods included: 10 years, Cross-section's data included: thirty-one firms, Total panel balanced observations: 310.

\section{4.(b) Panel data regression analysis}

Table 4.4, Model 4 was structure to investigate the influence of combination of three independent variable and control variables on corporate performance (ROTA, ROEQ \& ROCE) \& to deduce the key determinants of working capital management having the most significant influence on outcome variable 'corporate performance'. INVP, ARCP, CZ and SG are the most significantly variables affecting the firm's performance as ascertained by ROTA, ROEQ and ROCE. Model 4, the result revealed that the INVP, ARCP, and $\mathrm{CZ}$ are negatively associated with the firm's performance while $\mathrm{SG}$ exhibit a significant positive association, DR exhibited significant negative association with ROTA. Thus, results from the study of sample firms financial data advocate that firms have lower inventory turnover period, shorter receivable days and small size earns higher returns on its equity, capital employed and presents an efficient utilization of its economic resources. Moreover, faster inventory movements to customers and short receivable days enhances sales revenue thereby improving corporate performance. The model 4 -ROTA adjusted R2 is $70.4 \%$ ( $F$ statistics of 20.9) which infers that the changes in corporate performance (ROTA) was driven by WC variables considered in this model. The model 4 -ROEQ adjusted R2 is $40.99 \%$ (F statistics of 6.80 ) which infers that the changes in corporate performance (ROEQ) was driven by the Working capital (WC) variables considered in this model. The model 4 -ROCE adjusted R2 is $68.05 \%$ (F statistics of 18.79) which infers that the changes in corporate performance (ROCE) was driven by the Working capital (WC) variables considered in this model. The adjusted R2 of ROTA and ROCE revealed strong fit and ROEQ moderate fit. All the three models are acceptable as $p$-value $=.000$, indicating model 1 is well fit 
at 1 percent level of significance level.

\section{Conclusion}

The present study examined the influence on corporate performance by the determinants of Working Capital for a sample of thirty-one metal and mining firms listed on the National Stock Exchange (NSE) for financial years from 2009-2010 to 2018-2019 (10 years).

Working capital (WC) determinants were taken as an explanatory variable and performance as outcome variable. The results showed a significant negative influence of working capital determinants Accounts Inventory period (INVP)and Receivable Collection Period (ARCP) for a sample of Indian metal and mining firms on performance as measured by ROTA, ROEQ and ROCE. Indian metal and mining firms with shorter inventory period (INVP)and receivable Collection Period (ARCP) were able to generate higher profitability(ROTA, ROEQ \& ROCE). These results advocate that firm's respective managers can increase return on asset, increase in return on equity and increase in return on capital employed thereby creating value for firm's shareholders by reducing Inventory period (INVP)and Account Receivable Collection Period (ARCP) to an equitable minimum level. Efficient inventory and accounts receivable management by firm's management positively influence operational efficiency and eventually corporates performance. Results revealed a positive impact on the corporate performance of those firms that turned its inventory into sales in lesser days, resulting in decrease in holding costs and also mitigating the risk of inventory obsolescence. Thus, releasing the cash tied in inventory and using it efficiently for replenishment of inventory \& with reduced working capital cycle will result in efficient the utilization of resources, thus lowering the loan requirements \& the financial risks. Result also suggest that firms with lesser receivable collection days and with efficient collection of cash from receivables generates more earnings and at mitigating the risk of default/irrecoverable by receivables and liquidity problems.

No significant association was found between Accounts Payable Period (APP) and performance as ascertained by ROTA, ROEQ and ROCE. Result revealed a positive impact on corporate profitability of those firms that pays payables promptly as having shorter payable period, probably because firms availed the benefit of early payment discounts impacting positively the profit of the firm, and by avoiding any penalties of delay payment, moreover in future such firms may be aiming at improving its credit rating with suppliers to get higher credit period in future.

Regarding control variables result revealed a significant negative influence of $\mathrm{CZ}$ on ROTA, ROEQ \& ROCE. The smaller the corporate size, the higher the degree of efficient utilization of working capital resulting in increase in profit will be. Moreover, the result revealed the lower the total debt to total asset higher the return on assets and return on equity, firms that relied less on debts may be due to high finance cost and respective business's financial risk were able to generate higher returns. Sales Growth (SG) result indicated a significant positive influence on ROTA, ROEQ \& ROCE. As evident from correlation \& regression result, a shorter INVP,ARCP\& APP period brings an increase in sales, eventually resulting in increase in corporate performance.

The finding of research results may be useful for the financial managers in formulating systematic and efficient Working capital (WC) policies as to mitigate the financial risk and to improve the overall corporate performance and enhancing shareholders wealth .Research findings may also be useful for investors, researchers, and consultants.

\section{Limitations of This Research}

i. The sample size used in this research may not be sufficient to represent the entire industry. This study is restricted to 'metal and mining' sector with a sample of firms listed on the NSE, India for ten years from 2009-2010 to 2018-2019.Other sector companies are not considered. No significant association could be identified between corporate performance (ROTA,ROEQ \& ROCE) and account Payable (AP). Future research can be undertaken with larger sample size with comparative study of different industry or with comparative study of WCM of large and small and medium scale firms or comparative study country wise and with a longer study time frame, to say 15 or 20 years and result can be presented more effectively.

ii. The study has considered only annual secondary data (historical data in nature) to achieve the objectives which may not predict the marked influence on corporate performance with certainty. So, a further study can be initiated including other sectors or comparing other sectors using quarterly data for more accurate forecasting/ trend analysis to gauge the influence of WC on corporate performance.

iii. Lastly, the accounting measures of performance were applied with reference to internal factors and the limited number of variables was considered. Thus, future research can be conducted on for performance measures of performance (market value added (MVA), economic value added (EVA)) and external factors such as financial threat of competitive and economic factors were not included. 


\section{REFERENCES}

[1] Sharma, A, K., \& Kumar, S. (2011).Effect of working capital management on firm profitability empirical evidence from India. Global Business Review, 12(1), 159-173, 2011.

[2] Kajola, S,O., Nwaobia, A., Adedeji, S, B.(2014).Working capital management and firm performance :evidence from Nigerian listed firms. The International Journal of Humanities \& Social Studies, 2(4), 121-129, 2014.

[3] Chowdhury, A,Y., Alam, M, Z., Sultana, S., Hamid, M, K. (2018). Impact of working capital management on profitability: A case study on pharmaceutical companies of Bangladesh. Journal of Economics, Business and Management, 6(1), 27-35, 2018.
[4] Salman, A, Y., Folajin, O, O., Oriowo, A, O. (2014).Working capital management and profitability: A Study of selected listed manufacturing firms in Nigerian Stock Exchange. International Journal of Academic Research in Business and Social Sciences, 4(8),287-295, 2014.

[5] Kasozi, J. (2017). The effect of working capital management on profitability: a case of listed manufacturing firms in South Africa. Investment management and financial innovations, 14(2), 336-346, 2017.

[6] Makori, M, D., \& Jagongo, A. 2013.Working Capital Management and Firm Profitability: Empirical Evidence from Manufacturing and Construction Firms Listed on Nairobi Securities Exchange, Kenya. International Journal of Accounting and Taxation, 1(1), 2336-3121, 2013.

[7] https://www.ibef.org/industry/metals-and-mining.aspx. 